\title{
RESEARCH PROGRESS OF SUPERSONIC LASER DEPOSITION TECHNOLOGY
}

\author{
J IANHUA YAO ${ }^{1,2}$ and V. KOVALENKO ${ }^{1,2,3}$ \\ ${ }^{1}$ Research Center of Laser Processing Technology and Engineering, \\ Zhejiang University of Technology, Hangzhou, China \\ ${ }^{2}$ Zhejiang Provincial Collaborative Innovation Center \\ of High-end Laser Manufacturing Equipment, Hangzhou, China \\ ${ }^{3}$ Laser Technology Research Institute, NTUU «Kiev Polytechnic Institute», Kiev, Ukraine
}

\begin{abstract}
Supersonic laser deposition is a new coating and fabrication process, in which a supersonic powder stream generated in cold spray impinges onto a substrate which is simultaneously irradiated with a laser. It will be increasingly employed for depositing coatings and metal additive manufacturing because of its unique advantages: solid-state deposition of dense, homogeneous and pore-free coatings onto a range of substrate, high build rate at reduced operating costs without the use of expensive gas heating and large volumes of helium, and opening up a new opportunity for efficiently depositing high hardness metallic powders which are usually difficult to be deposited solely by cold spray. Based on the current research results in our group, this paper systematically reviews state-of-the-art of supersonic laser deposition technique at home and abroad, from the viewpoints of materials selection, process optimization, properties characterization, equipment design and so on. The existing issues in these aspects are deeply analyzed, and the corresponding solutions are tentatively proposed. Meanwhile, the potential industrial applications of supersonic laser deposition in various fields are elaborated in detail, as well as the future perspectives and challenges facing this technology, in order to provide insight for further investigations and innovation in supersonic laser deposition as an emerging combination additive re-manufacturing technology with high efficiency, low cost and high quality. 16 Ref., 18 Figures.
\end{abstract}

Keywor $\boldsymbol{d} \boldsymbol{s}$ : supersonic laser deposition, materials, process parameters, performances, applications

Supersonic laser deposition (SLD) is a newly developed technology in the field of laser material processing, which can be used for surface modification and coating of engineering components for increased functionality [1-3]. This technology combines the supersonic powder beam found in cold spray (CS) with laser heating of the deposition site. In SLD, a laser heats both the spraying particles and the substrate to $30-80 \%$ of their melting point, thus significantly reducing the strength of the particles and substrate, and allowing the particles to plastically deform and build up a coating at an impact velocity about half of that in CS.

SLD technology has been increasingly employed for coating deposition because of its technological and economic advantages over conventional coating methods, namely solid-state deposition of dense, homogeneous and pore-free coatings onto a range of substrates; high deposition rate at reduced operating costs without the use of expensive heating and process inert gases; less sensitivity to feedstock materials characteristics; consolidation of difficultto-deposit powders; and significant improvements in the properties of coating materials. More importantly, lower processing temperatures and shorter processing time of SLD technique will enable the coating, and fabrication of near-net shape components with little or no melting, thus avoiding the deleterious effects of high-temperature processes such as laser cladding and

(C) JIANHUA YAO and V. KOVALENKO, 2016 traditional thermal spray processes include depositsubstrate dilution, high thermally induced residual stresses, and as-solidified microstructures, which lead to component distortion and poor mechanical properties.

As compared to CS, the inclusion of laser heating into SLD can considerably softened the spraying particles and the substrate, which would reduce the critical deposition velocity and allow bonding to occur on impact at velocities around half those found in $\mathrm{CS}$, even when depositing materials that are difficult to process solely using CS. Eliminating the need for high impacting velocities permits cold or slightly heated nitrogen to be used instead of high-temperature helium as the process gas, thus reducing operating costs by over an order of magnitude. This reduction in capital and operating costs means that SLD may be viable in many applications, for which CS has proved too costly allowing the fully solid process route found in CS to find a use in a wider range of applications. A variety of material coatings such as $\mathrm{Cu}$, Ti, Stellite 6 , $\mathrm{Ni60}, \mathrm{Al}-\mathrm{Cu}$ and Al-Si alloys, have been successfully prepared with SLD technique [4-16].

In order to provide insight for further investigations and innovation in SLD as an emerging combination additive re-manufacturing technology with high efficiency, low cost and high quality, this paper presents a systematical overview about state-of-theart of SLD technology based on the current research 
results in our group, from the viewpoints of equipment design, materials selection, process optimization, properties characterization, and so on. The existing issues in these aspects are deeply analyzed and the corresponding solutions are tentatively proposed. Meanwhile, the potential industrial applications of SLD in various fields are elaborated in detail as well as the future perspectives and challenges facing this technology.

Supersonic laser deposition system. Schematic diagram of the SLD system is illustrated in Figure 1, $a$. The laser energy and powder distribution are schematically illustrated in Figure 1, $b$. High-pressure gas was supplied to a converging-diverging nozzle in two different imports: one was through the gas heater, the other was via a powder feeder, where feedstock powders were held. The feedstock powder stream and high-pressure gas were mixed and passed through the nozzle, where the particles were accelerated to supersonic speed. The gas heating temperature, gas pressure and powder feeding rate were monitored and adjusted by the control unit of the CS equipment (Figure 2,a). The high-velocity particles impacted a region of the substrate, which was synchronously heated by a diode laser (Figure 2, $b$ ). Combined lenses were used to focus the laser beam onto the substrate surface. A high-speed infrared pyrometer was used to obtain real-time temperature measurements and control the temperature of the deposition zone during the SLD process. Data from the pyrometer was fed through a closed-loop feedback system, which altered laser power as necessary to maintain the desired temperature. The nozzle, laser head and pyrometer were assembled on a robot (Figure 2,c). The spraying nozzle was perpendicular to the substrate surface. The laser beam was at $30^{\circ}$ angle to the surface normal. In deposition process, the substrate was stationary and the nozzle, laser head and pyrometer were moveable, controlled by the robot. The process gas can be compressed air (or high-pressure nitrogen), which can be provided by an air compressor (or a manifolded cylinder palette) (Figure 2, $d$ ).

Coating fabrication and characterization. Single material coatings. This section focuses on the comparison of the single material coatings prepared by SLD and other conventional coating technologies such as CS and laser cladding, in regard to deposition efficiency (DE), coating density, microstructure evolution, interfacial bonding, properties, etc.

Comparison of single material coatings prepared by SLD and CS. Shown in Figure 3 is the comparison of thickness of CS-Cu and SLD-Cu coatings. It is evident that the SLD-Cu coating is thicker than the $\mathrm{CS}-\mathrm{Cu}$ coating. The peak thickness of CS-Cu coating is around $1.3 \mathrm{~mm}$ while that of SLD-Cu coating is about $2.2 \mathrm{~mm}$, i.e laser irradiation increased the peak
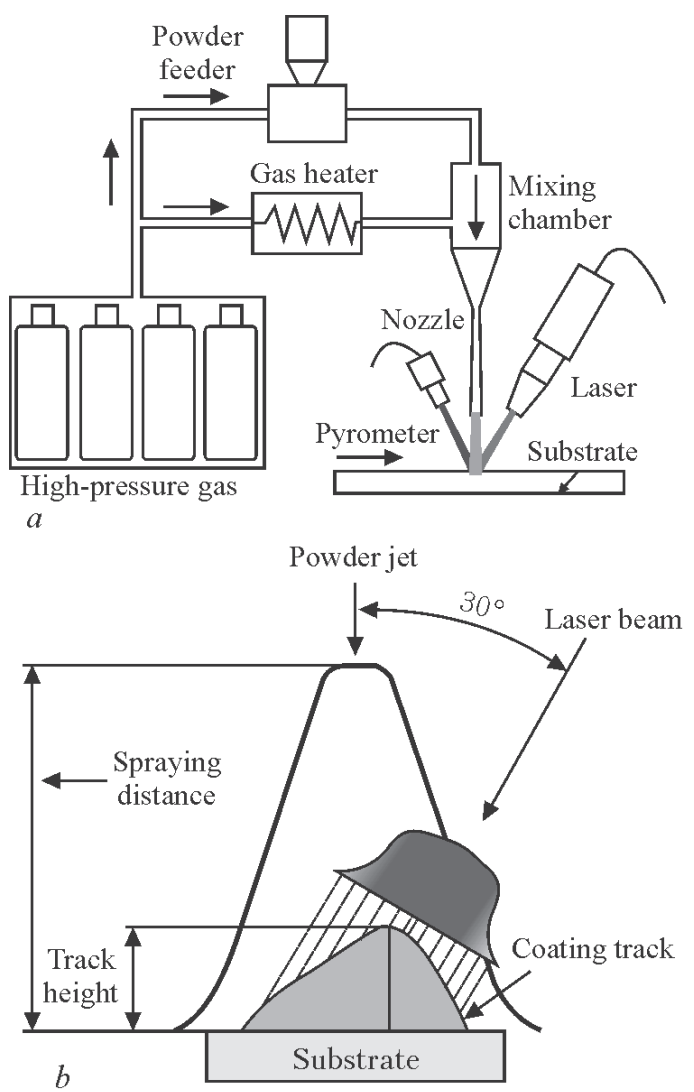

Figure 1. Schematic illustration of SLD system $(a)$, and laser energy and powder distribution in SLD process $(b)$

coating thickness by $70 \%$. In other words, laser heating considerably improved the DE.

Figure 4 shows the comparison of density of CS$\mathrm{Cu}$ and SLD-Cu coatings. It is observed that CS$\mathrm{Cu}$ coating has lots of gaps and pores between the deformed copper particles, while the SLD one has a much denser microstructure, with gaps and pores hardly observed. Porosity measurements using image analysis software indicated that the porosity of the

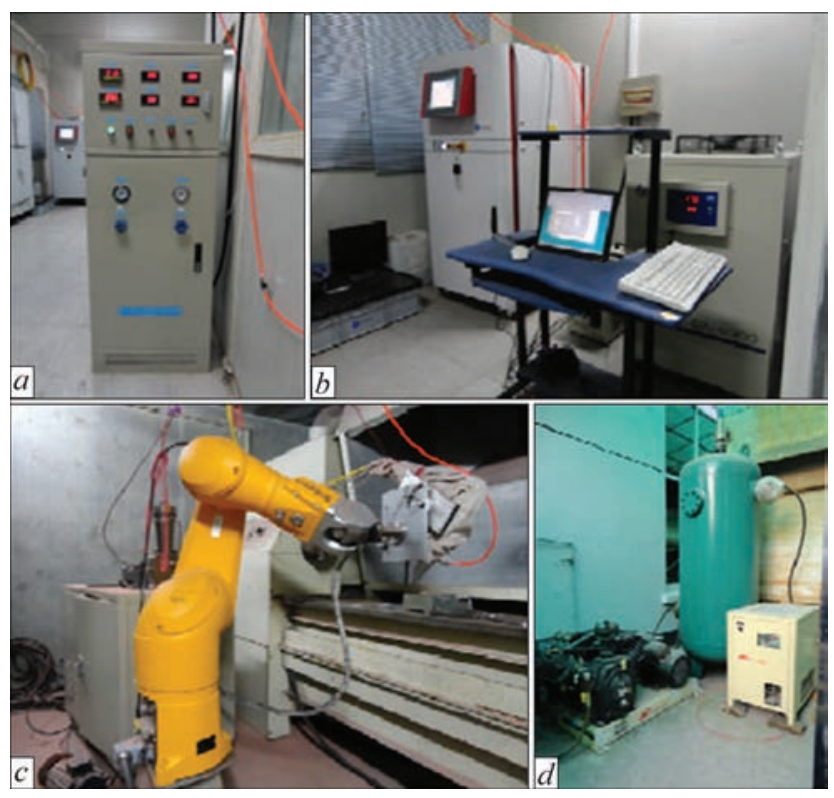

Figure 2. SLD system: $a-$ control unit of SC equipment; $b$ diode laser; $c$ - robot; $d$ - air compressor 

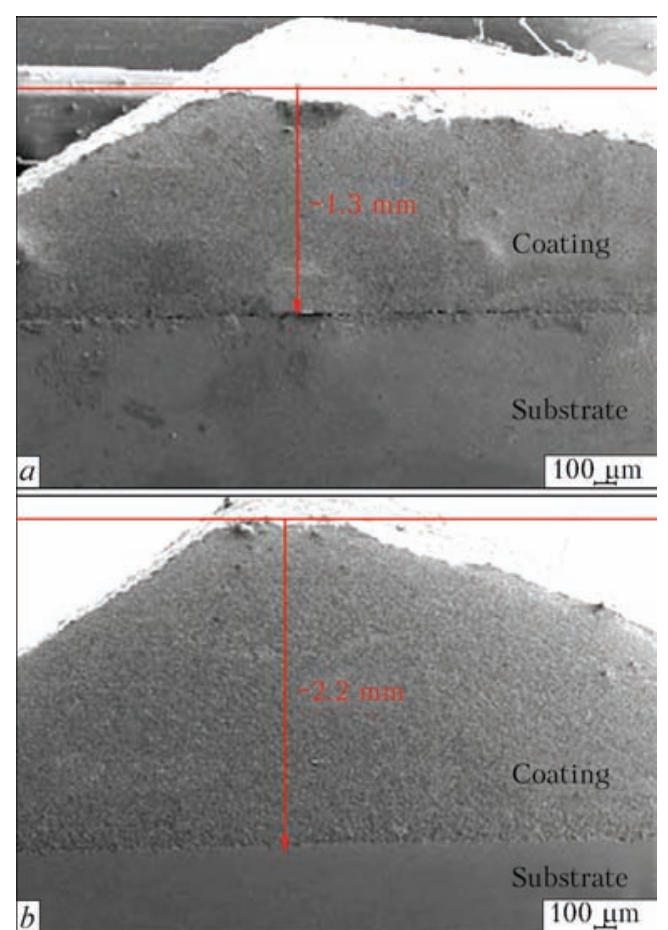

Figure 3. Comparison of the $\mathrm{CS}-\mathrm{Cu}(a)$ and SLD-Cu $(b)$ coating thickness

CS-Cu coating was $3.367 \%$ in area, while it was only $0.08 \%$ in area for the SLD-Cu coating. This confirms the beneficial effect of laser irradiation on the coating density.

Figure 5 presents the coating-substrate interfacial bonding of the $\mathrm{CS}-\mathrm{Cu}$ and SLD-Cu coatings. As can be seen from Figure 5, a, there is an obvious crack observed at the interface between coating layer and substrate of the CS-Cu coating specimen, but this is not found in the SLD-Cu coating, instead, material penetration has occurred at the interface of this coating

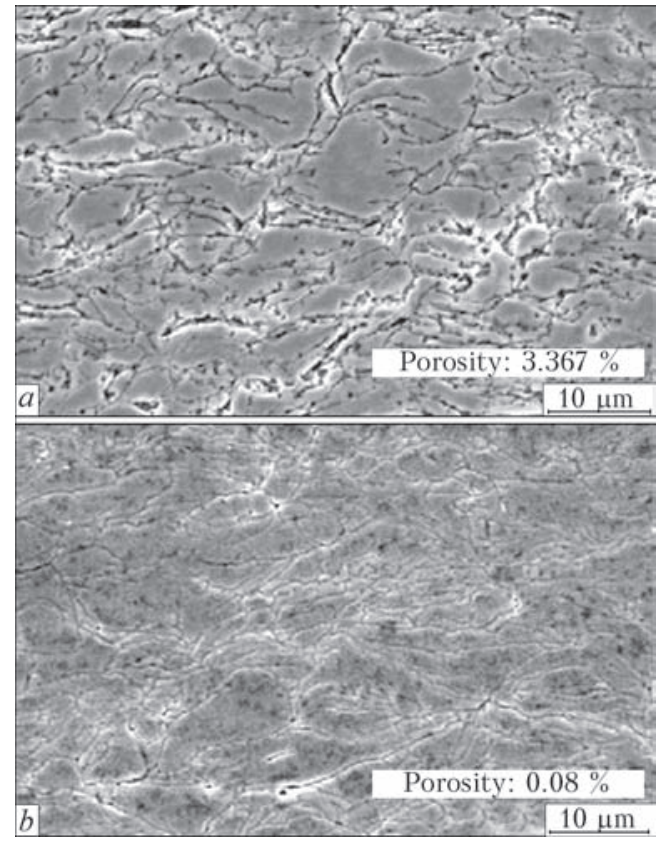

Figure 4. Comparison of density of the CS-Cu $(a)$ and SLD-Cu (b) coatings
(Figure 5, b), which would enhance the coating bonding to the substrate. Adhesion strength test according to ASTM Standard C633 was performed on the CS-Cu and SLD-Cu coatings in order to quantify the real bonding force for each coating. The comparison of adhesion strength of coatings is shown in Figure 5, $c$. It can be seen that the adhesion strength of the CS-Cu coating is very weak but it increased significantly with the assistance of laser irradiation.

From the above-mentioned results it can be concluded that DE, coating density and interfacial bonding of CS coating can be improved by the assistance of laser irradiation. The improvement of DE should be ascribed to the reduction of critical deposition velocity due to the softening of spraying particles by laser heating. One of the most important parameter in CS process is the critical deposition velocity. For a given material, there exists a critical deposition velocity that must be achieved. Only
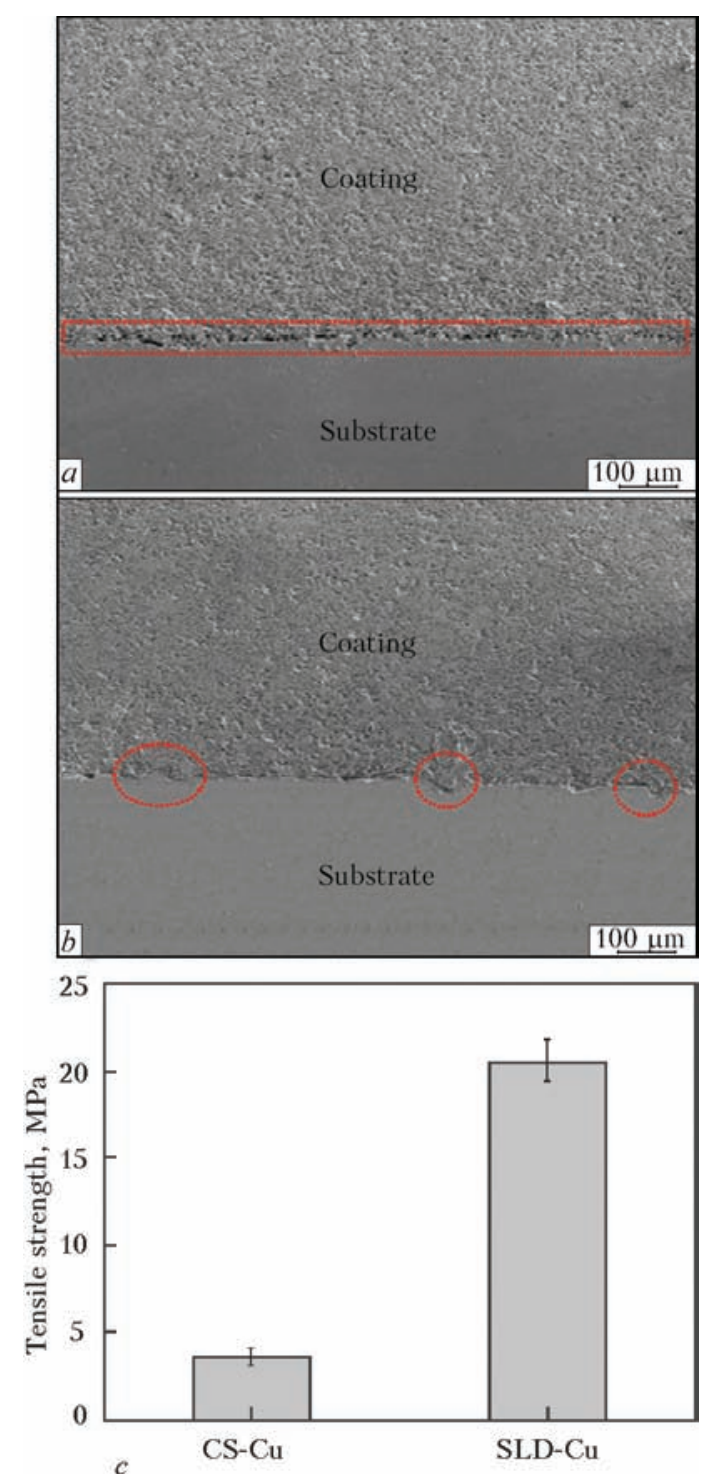

Figure 5. Coating substrate interfacial bonding of the CS-Cu (a) and SLD-Cu (b) coating, and comparison of their adhesion strength $(c)$ 


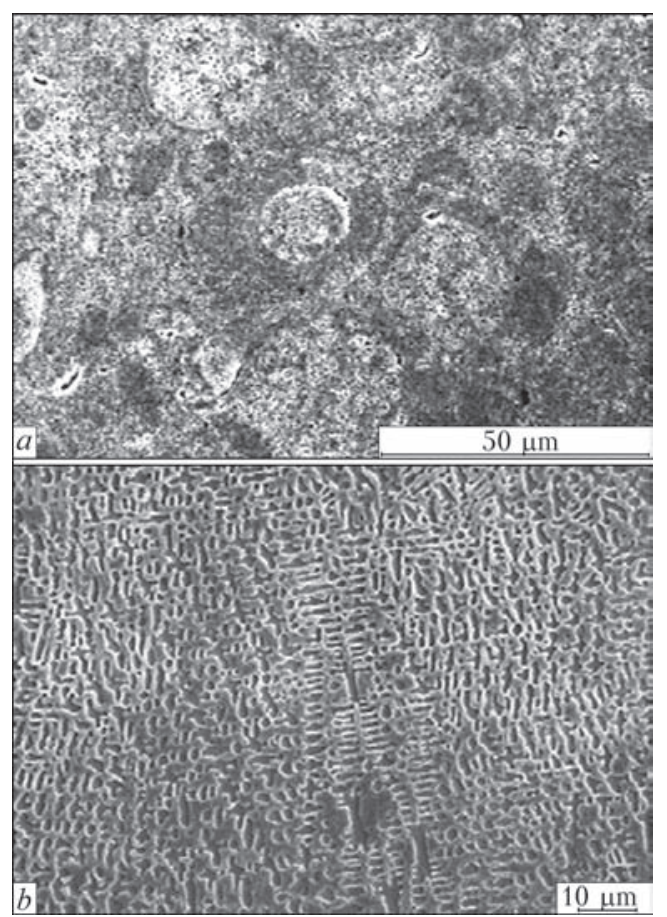

Figure 6. Comparison of microstructure of the SLD-Ni60 $(a)$ and LC-Ni60 (b) coatings

particles, whose velocities exceed this value, can be effectively deposited, in turn producing the desired coating. Conversely, particles that have not reached this threshold velocity contribute to the erosion of the substrate. Theoretical modelling of critical deposition velocity $v_{\text {cr }}, \mathrm{m} / \mathrm{s}$, proposed by Assadi et al. can be expressed as

$$
v_{\text {cr }}=667-14 \rho+0.08 T_{\mathrm{m}}+0.1 \sigma_{\mathrm{u}}-0.4 T_{\mathrm{i}},
$$

where $\rho$ is the material density $\left(\mathrm{g} / \mathrm{cm}^{3}\right) ; T_{\mathrm{m}}$ is the melting temperature $\left({ }^{\circ} \mathrm{C}\right) ; \sigma_{u}$ is the ultimate strength (MPa); and $T_{\mathrm{i}}$ is the initial particle temperature $\left({ }^{\circ} \mathrm{C}\right)$. According to formula (1), particle preheating will decrease the critical deposition velocity because as $T_{\mathrm{i}}$ is increased, $\sigma_{\mathrm{u}}$ of materials is reduced. Both the increase of $T_{\mathrm{i}}$ and reduction of $\sigma_{\mathrm{u}}$ would contribute to decrease of $v_{\text {cr. }}$

In SLD, the powder jet and laser beam partially overlapped with each other. Although the spraying particles were travelling at high velocities and had limited time of exposure to the laser, it is expected that the particles would be significantly heated in flight by laser prior to hitting the substrate because of the high laser power density and small particle size, which could bring down the critical deposition velocity of spraying particles. As a consequence, the proportion of particles exceeding this velocity would increase, leading to the DE improvement. In CS process, the initially deposited particles are hammered by successive highspeed impacting particles. The softened particles by laser heating get easily deformed by the impact of particles at a high velocity, leading to tight bonding of deposited particles (high coating density). In the case of synchronous laser irradiation on the deposition

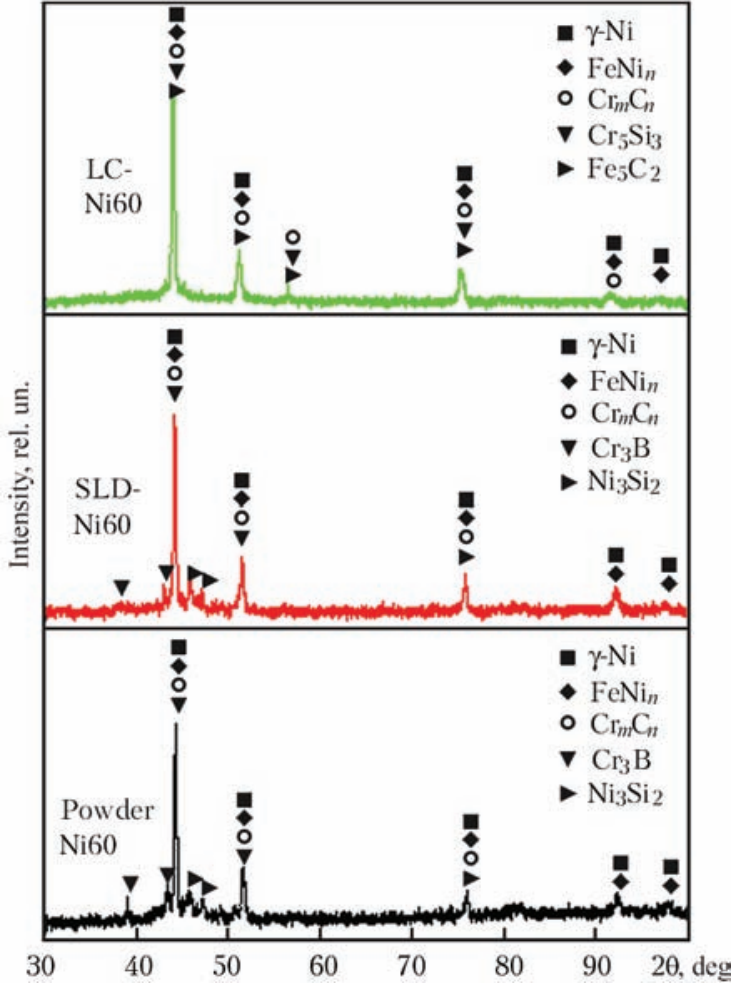

Figure 7. XRD patterns of the LS-Ni60 and SLD-coatings and Ni60 powder

site, the substrate temperature is increased and it is thereby softened. The softened substrate easily lodges the particles to form mechanical interlocking. Moreover, the increased substrate temperature can promote the atomic diffusion between the coating and the substrate, which greatly increases the possibility of metallurgical bonding. All these contribute to the good interfacial bonding of the SLD coating.

Comparison of the single material SLD and laser clad coatings. Figure 6 shows the SEM microstructure of the Ni60 coating specimens prepared with SLD and LC. As can be seen, the microstructure of the SLDNi60 coating shows accumulated plastic deformation of the Ni60 particles with the similar fine as-cast structure to original powder particles (solid-state deposition), while the microstructure of the LC-Ni60

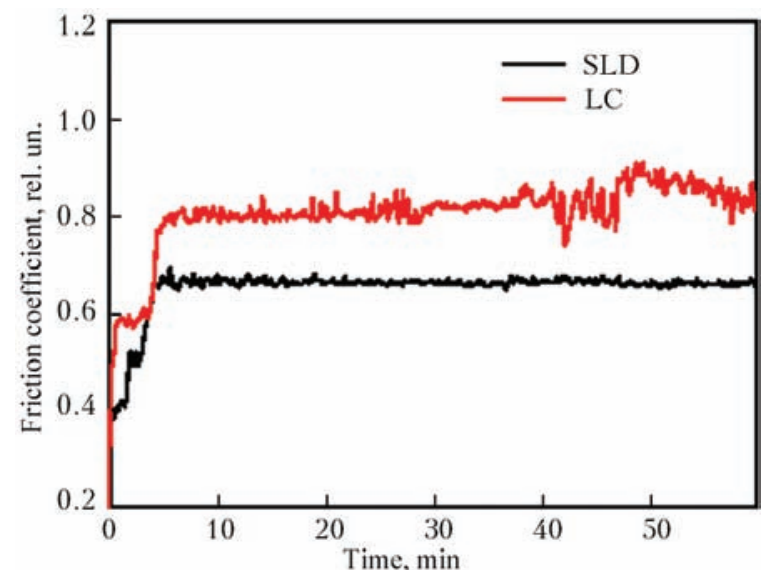

Figure 8. Friction coefficient versus sliding time for SLD-Ni60 and LC-Ni60 coatings 

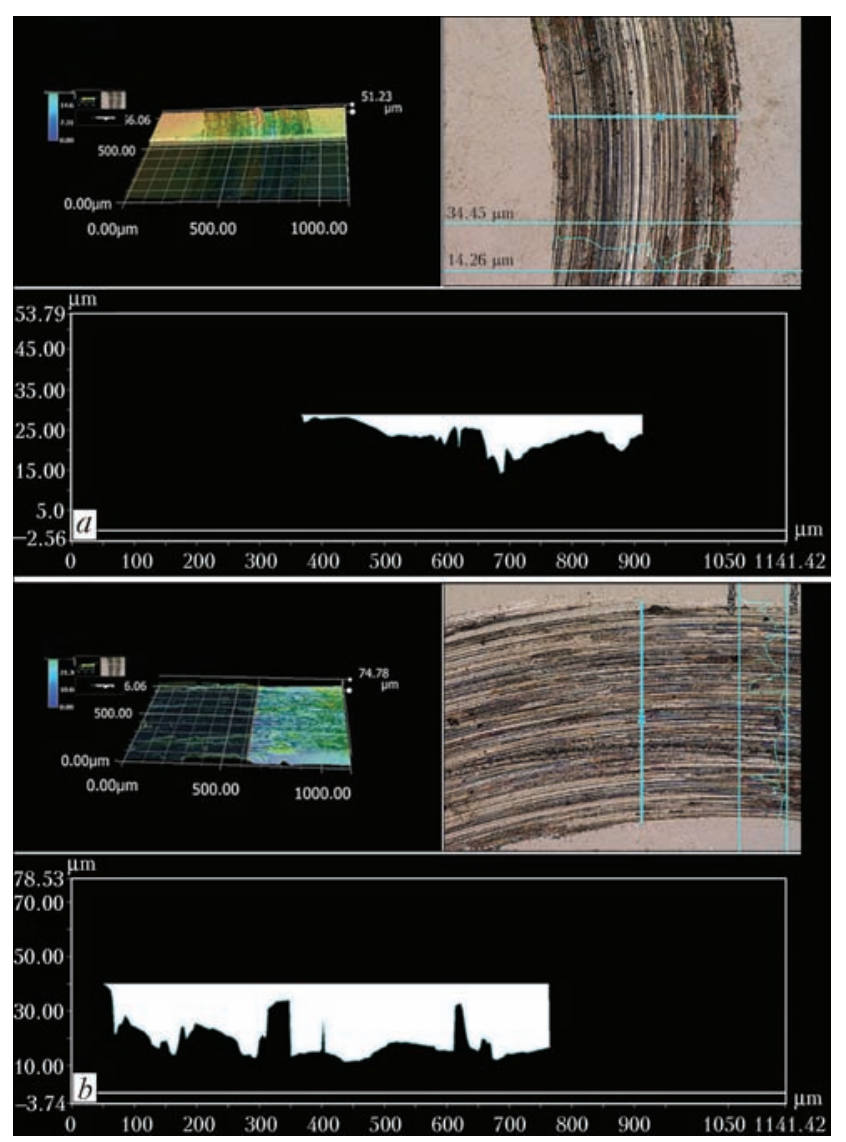

Figure 9. Cross-sectional profile of wear track for SLD-Ni60 (a) and LC-Ni60 (b) coatings

coating exhibits a typical coarse cladding dendritic structure.

Further XRD analysis (Figure 7) shows that the SLD-Ni60 coating has identical phases to that of the original powder particles. However, the phases in XRD pattern of the LC-Ni60 coating differ from those of the SLD-Ni60 coating and Ni60 powder, in that $\mathrm{LC}$ process generated a new phase $\mathrm{Fe}_{5} \mathrm{C}_{2}$ due to dilution effect. Shown in Figure 8 are the evolutions of friction coefficient of the coating specimens that recorded during the wear test. As illustrated, the

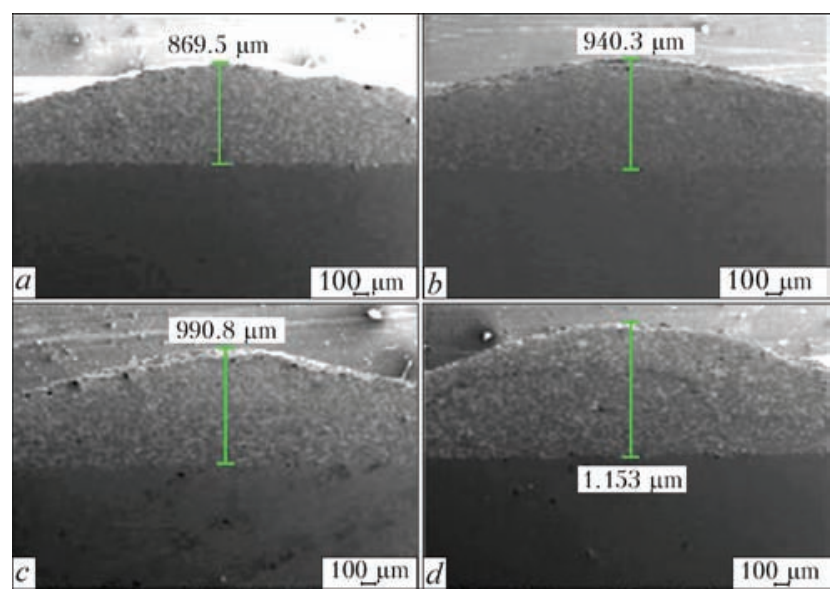

Figure 10. Thickness of the WC/SS316L composite coating deposited without laser heating $(a)$, at $500(b), 700(c)$ and $900(d)$ ${ }^{\circ} \mathrm{C}$ deposition temperature friction coefficient of the SLD specimen is much smaller and more stable than that of the LC specimen. Deep plough scars can be observed obviously in the wear track of the LC specimen, while the SLD specimen looks smoother (Figure 9). Also, the wear track width of the LC specimen is wider than that of the SLD one. It is clear that the SLD coating has better wear resistance than the LC one.

Laser irradiation in SLD provides heat, which can synchronously soften the high-speed particles and substrate, while the heat in LC process melts the particles. Since SLD process has less laser energy input than LC one, the coating-substrate interface and HAZ of the SLD specimen are smoother and smaller. Moreover, due to the relatively low temperature in SLD, the as-deposited Ni60 coating still remains the same microstructure and phases as that of the feedstock powder materials. The superior wear resistance property of the SLD-Ni60 coating to the LC one should be attributed to the finer structures in the SLD coating, that is, the carbides and borides are more homogeneously distributed in the nickel matrix. It should be noted that hard Ni60 powder particles can be successfully deposited by SLD while it is impossible to be deposited by CS, indicating the SLD technique broadens the materials range that can be processed with CS. Furthermore, this novel deposition technique surpasses conventional LC technique, when used to deposition of hard materials such as Ni60 alloy, in that it can suppress the dilution of the steel substrate.

Metal-matrix composite material coatings. Comparisons of metal-matrix composite (MMC) material prepared by SLD and other conventional coating technologies are focused on the heat sensitive materials such as tungsten carbide (WC) and diamond.

Comparison of the SLD- and CS-MMC coatings. Shown in Figure 10 are the SEM images of cross-section of the WC/SS316L composite coating specimens. As observed, the central peak height strongly depends on the laser heating temperature. The peak height of the WC/SS316L coating deposited without laser assistant is $869.5 \mu \mathrm{m}$, and it is gradually increased to 1.153 $\mathrm{mm}$ with the increase of deposition temperature from 500 to $900{ }^{\circ} \mathrm{C}$. This result indicates that laser heating can also improve the $\mathrm{DE}$ of MMC coatings as similar to the single material coatings, which is ascribed to the reduction of critical deposition velocity by laser irradiation. It can be found in Figure 11 that WC particles are evenly distributed in all the specimens, and the concentration of $\mathrm{WC}$ particles in the coatings is increased with deposition temperature. SEM images at high magnification show (Figure 12) that WC particles are not effectively embedded into the SS316L matrix in the case without laser heating. With less laser heating or at low deposition temperature, 

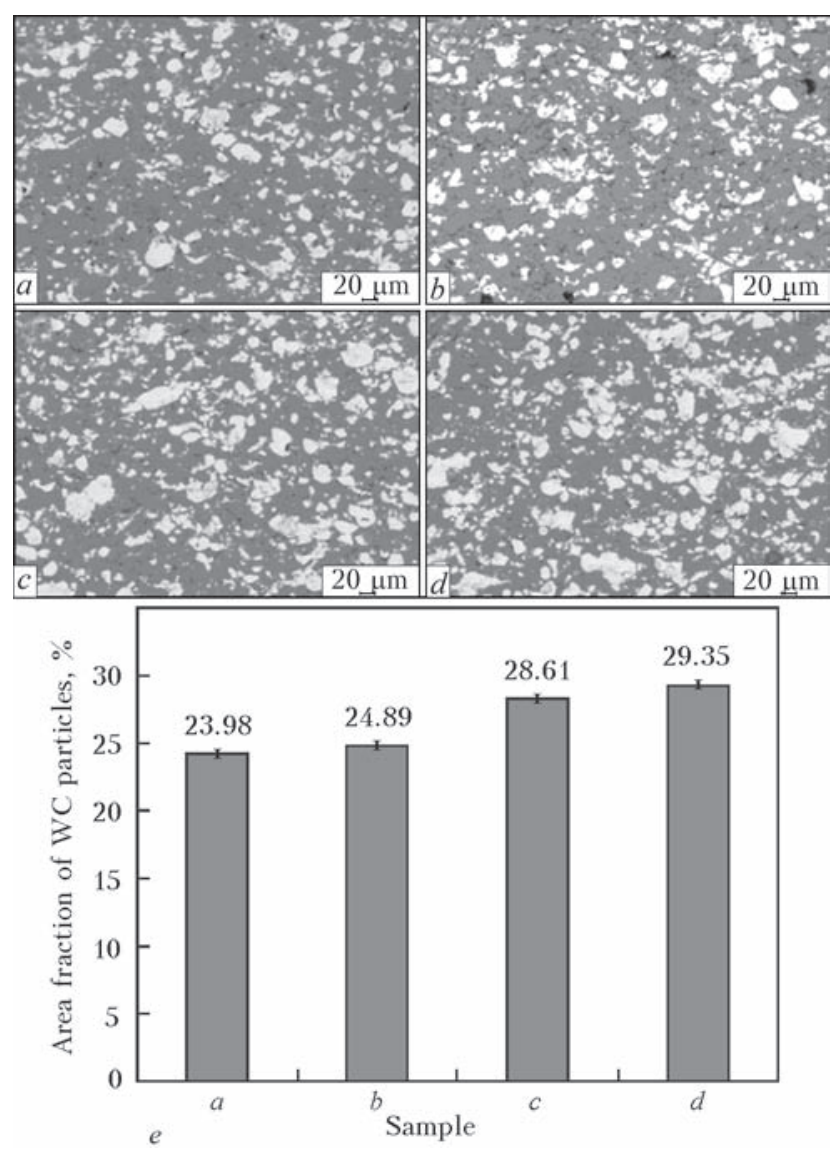

Figure 11. Distribution and concentration of WC particles in the composite coatings produced without laser heating $(a)$, at $500(b)$, $700(c)$ and $900(d){ }^{\circ} \mathrm{C}$ deposition temperature; $e$ - area fractions although the WC particles can be embedded in the coating, obvious gaps can still be found at the interface between WC particles and SS316L matrix. Further increasing deposition temperature enhances the interface bonding strength. The WC particles are well embedded in the SS316L matrix with little gaps.

The beneficial effects of laser irradiation on the concentration of WC particles in the WC/SS316L composite coatings can be attributed to the softening of SS316L powder. During SLD process, the WC

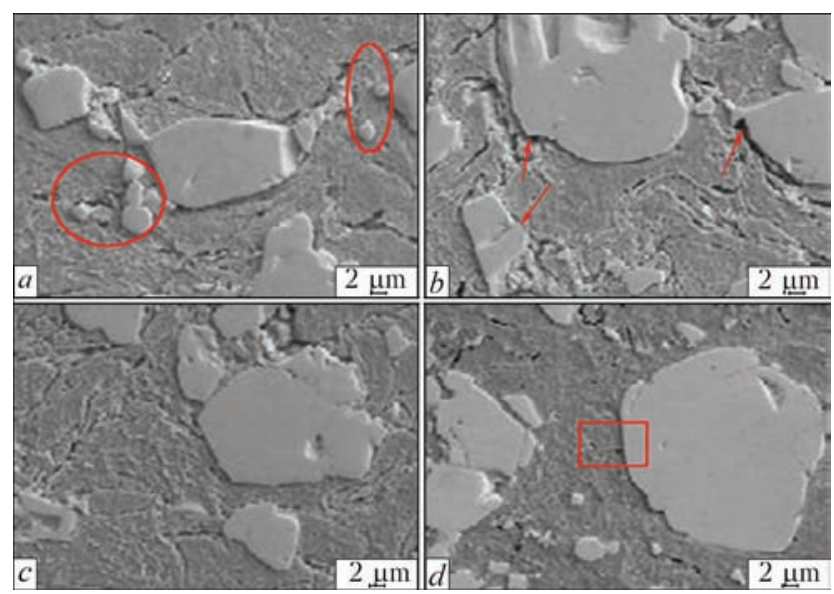

Figure 12. Interface bonding between SS316L matrix and WC particles in coatings deposited without laser heating $(a)$, at 500 (b), $700(c)$ and $900(d){ }^{\circ} \mathrm{C}$ deposition temperature

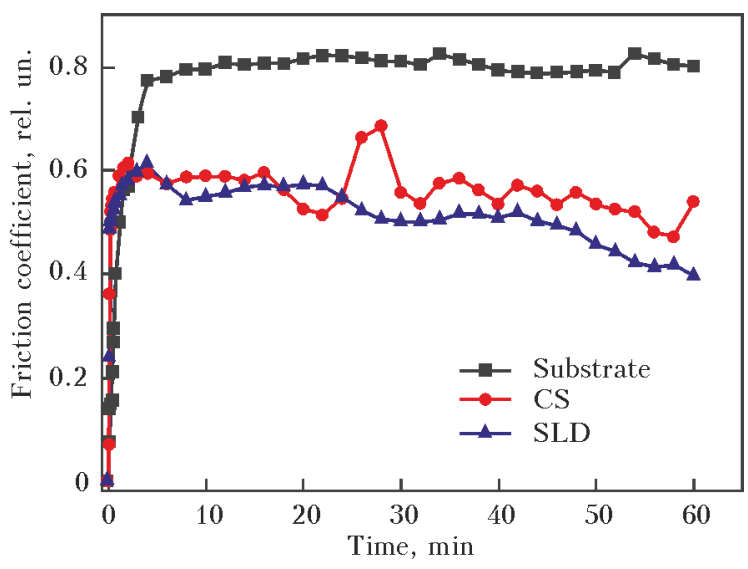

Figure 13. Friction coefficient versus sliding time for SLD- and CS-WC/SS316L composite coatings

particles may not deform due to high hardness and they are embedded in the deformable SS316L matrix. Without laser assistance or with less laser heating, SS316L powder may not be softened enough to accommodate the hard particles, resulting in relatively low $\mathrm{WC}$ concentration. In the case of more laser heating, SS316L powder easily deforms to take in $\mathrm{WC}$ particles owing to sufficient softening, leading to higher WC particle concentration. Furthermore, due to softening effect by laser irradiation in SLD, SS316L powder particles are easier to deform, which also favours lodging the impacting $\mathrm{WC}$ particles to form intimate bonding, therefore showing improved bonding strength than the CS coating. The relatively high content of WC particles in the composite coating and the strong interfacial bonding between WC particles and SS316L matrix results in better

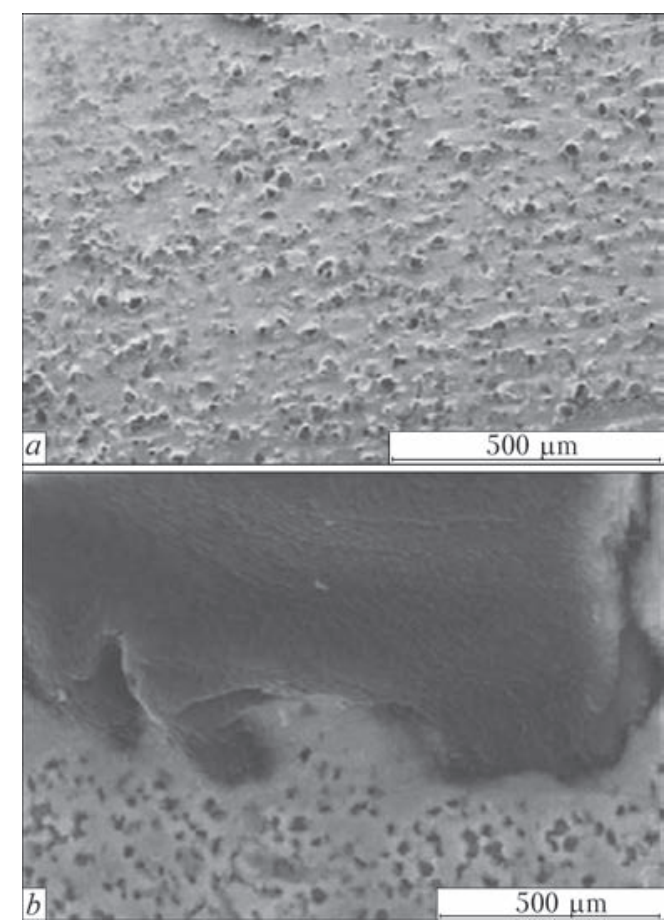

Figure 14. Microstructure of the SLD-diamond/Ni60 composite coating 


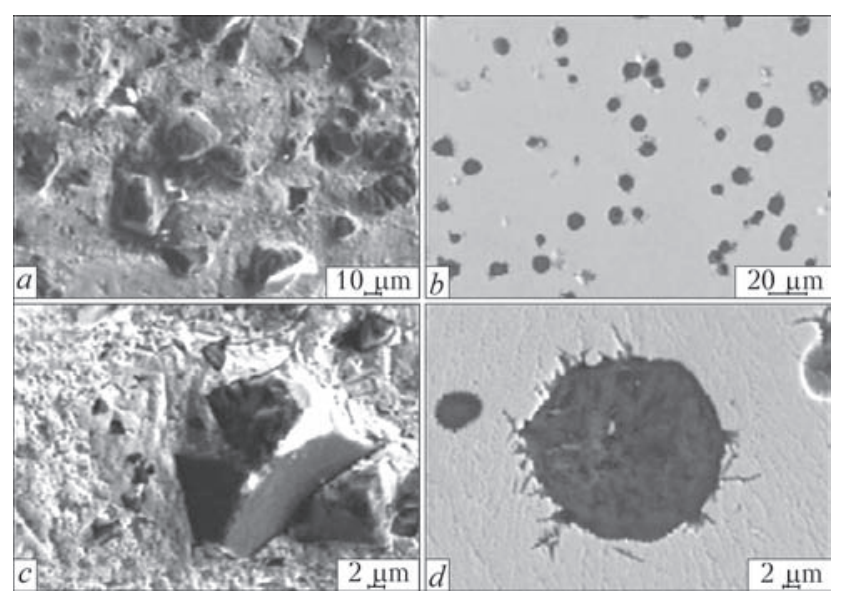

Figure 15. Graphitization of diamond in the SLD- $(a, c)$ and LCdiamond/Ni60 coatings $(b, d)$

tribological properties of the WC/SS316L coating produced by SLD than with CS, as shown in Figure 13.

Comparison of SLD- and LC-MMC coatings. Figure 14 presents the microstructure of the diamond/ Ni60 composite coating produced with SLD. It shown that the diamond particles are uniformly distributed within the Ni60 matrix (Figure 14,a), and the diamond particles are firmly embedded in the Ni60 matrix with good interface bonding (Figure 14, b). Most of the diamond particles in the composite coatings were fully retained. This may be due to the softened Ni60 matrix by laser heating. In this case, Ni60 particles were more prone to deform by adiabatic shear instability. The softened Ni60 particles are beneficial for wrapping and holding the diamond particles.

Figure 15 provides some information on diamond graphitization between the SLD-diamond/Ni60 and the LC-diamond/Ni60 coatings. In LC process, the high temperature and oxidation environment of molten pool, produced by laser irradiation, make the diamond particles easier to graphitize, compared with SLD process. In Figure 15, $b$ and $d$, the black regions indicate serious graphitization of the diamond particles; carbon diffusion occurred at the interface between the diamond particle and Ni60 matrix. It is

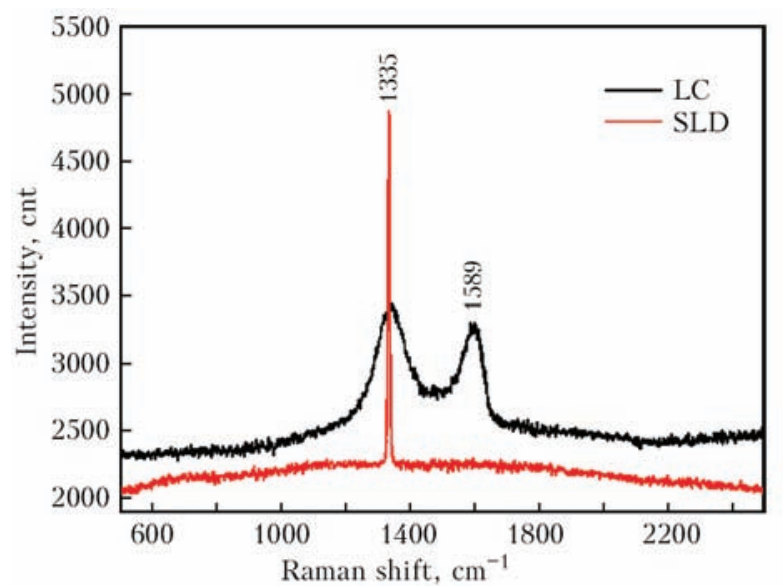

Figure 16. Raman spectra of the SLD- and LC-diamond/Ni60 coatings

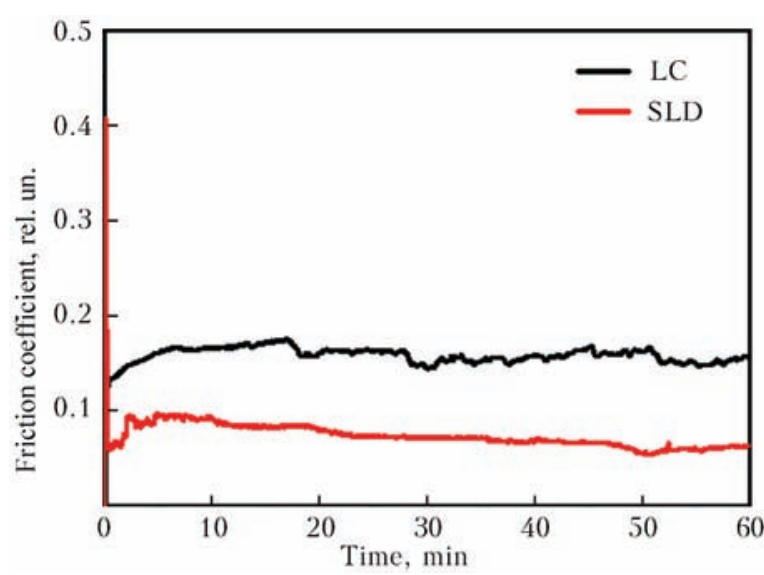

Figure 17. Friction coefficient versus sliding time for LC- and SLD-diamond/Ni60 coatings

also found that the irregular shape of the diamond particles was changed to spherical shape during LC. However, graphitization is not as severe in the SLD coating specimen as in the LC one.

From the Raman spectra in Figure 16 it can be seen that not only a typical diamond peak at $1335 \mathrm{~cm}^{-1}$ but also an obvious non-diamond component peak at $1589 \mathrm{~cm}^{-1}$ are presented in the LC coating, while only a single and sharp diamond peak is observed at $1335 \mathrm{~cm}^{-1}$ in the SLD coating. The Raman spectra analysis results demonstrate that part of the diamond particles have been graphitized in the LC coating but no graphitization occurred in the SLD coating. All these findings suggest that the relatively high impact pressure, low deposition temperature and inert $\mathrm{N}_{2}$ atmosphere are beneficial for preventing diamond particles from graphitizing during SLD process.

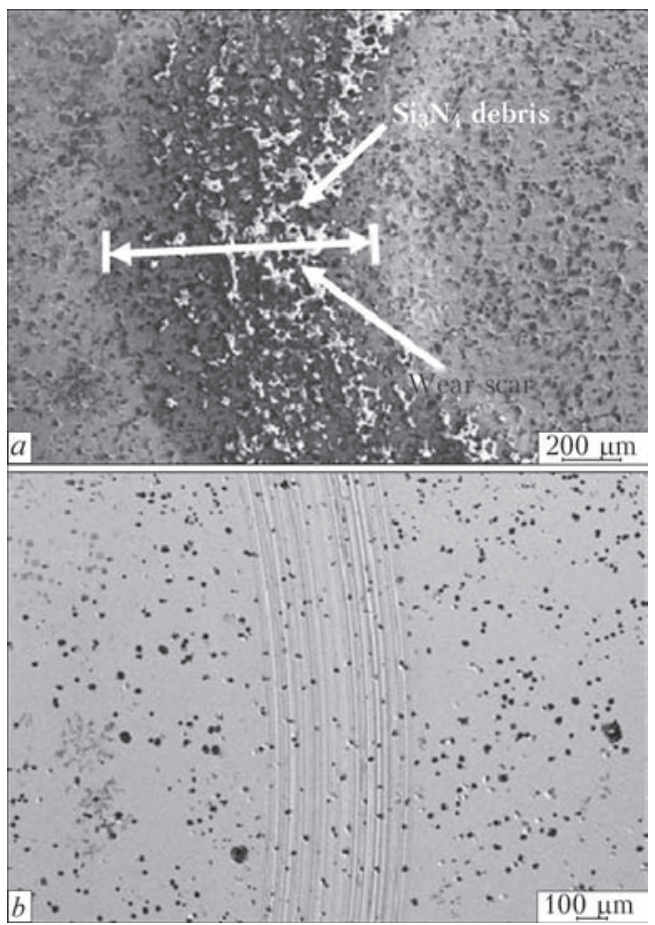

Figure 18. SEM images of worn surfaces: $a$ - wear track of SLD specimen; $b$ - LC specimen 
According to the results of wear test (Figures 17 and 18), the diamond/Ni60 composite coating has excellent tribological properties. Under the cyclic load in the wear process and the cutting of irregular diamond particles, the surface of the $\mathrm{Si}_{3} \mathrm{~N}_{4}$ grinding ball was seriously abrased. It is believed that friction between the contact surfaces was reduced due to abrasion resistance of hard irregular diamond particles. The low friction and high abrasion resistance of the diamond/Ni60 composite alleviate the damage of wear surface. Since the sliding wear mechanism of diamond is abrasion, the wear surface of pin is damaged by groove ploughing. The wear track is characterized by numerous discontinuous, short and shallow grooves. These imply that wear resistance of the SLD specimen is better than that of the LC specimen, because the interface bonding between the diamond and Ni60 particles is strong enough to sustain the mechanical attack under the wear.

Future perspective and challenges. On the basis of the results from the reviewed studies, it can be concluded that SLD has great potential for rapid transfer of laboratory-developed technology to various industrial fields such as automotive, marine, biomedical, aeronautical/aerospace, power generation, petrochemical, and mining. Furthermore, the need to reduce high cost of preparing coatings, reduce fabrication stages into a single step, and improve coating's functional properties are among the reasons that SLD technique will continue to gain attention both in academic and industrial research. Some application-oriented challenges such as specific tribology, severe abrasive wear, hightemperature creep, fatigue, and severe erosion need to be investigated for successful utilization of SLD technology in the industry.

The focus of further work should be on improving the efficiency and capability of SLD technology. The efficiency in current study was limited due to the mismatching of laser spot and the powder footprint and the non-uniform heat distribution (Gaussian heating profile) across the deposition site. Using a more powerful laser would enable the laser spot to be increased to the size of powder footprint, and the effects of deposition at higher traverse rates and build rates to be investigated. If a top-hat laser beam profile is used, a more uniform temperature distribution could be expected, which would increase consistency of deposition conditions across the tracks, thus improve process control. The use of higher particle velocities should be investigated through the use of gas heating or improved nozzle designs, as well as improved deposition efficiency, density and mechanical properties of coatings should be expected.

Acknowledgements. The authors would like to appreciate financial supports from the National Natural Science Foundation of China (51475429), the Zhejiang Provincial Commonweal Technology Applied Research Project (2014C31122) and the Postdoctoral Scientific Research Project of Zhejiang Province (Z42102002).

1. Bray, M., Cockburn, A., O’Neill, W. (2011) The laser-assisted cold spray process and deposit characterization. Surface and Coating Techn., 203, 2851-2857.

2. Lupoi, R., Sparkes, M., Cockburn, A. et al. (2011) High speed titanium coating by supersonic laser deposition. Materials Letter, 65, 3205-3207.

3. Jones, M., Cockburn, A., Lupoi, R. et al. (2014) Solid-state manufacturing of tungsten deposits onto molybdenum substrates with supersonic laser deposition. Ibid., 134, 295-297.

4. Olakanmi, E.O., Doyoyo, M. (2014) Laser assisted cold-spray corrosion- and wear-resistant coatings: A review. J. Thermal Spray Techn., 23, 765-785.

5. Kulmala, M., Vuoristo, P. (2008) Influence of process conditions in laser-assisted low pressure cold spraying. Surface and Coatings Techn., 202, 4503-4508.

6. Li, B., Yang, L.J., Li, Z.H. et al. (2015) Beneficial effects of synchronous laser irradiation on the characteristics of coldsprayed copper coatings. J. Thermal Spray Techn., 24, 836-847.

7. Luo, F., Cockburn, A., Lupoi, R. et al. (2012) Performance comparison of Stellite 6 deposited on steel using supersonic laser deposition and laser cladding. Surface and Coatings Techn., 212, 119-127.

8. Yao, J.H., Yang, L.J., Li, B. et al. (2015) Beneficial effects of laser irradiation on the deposition process of diamond/Ni60 composite coating with cold spray. Appl. Surface Sci., 330, 300-308.

9. Li, B., Yao, J.H., Zhang, Q.L. et al. (2015) Microstructure and tribological performance of tungsten carbide reinforced stainless steel composite coatings by supersonic laser deposition. Surface and Coatings Techn., 275, 58-68.

10. Yao, J.H., Yang, L.J., Li, B. et al. (2015) Characteristics of performance of hard Ni60 alloy coating produced with supersonic laser deposition technique. Materials and Design, 83, 26-35.

11. Olakanmi, E.O., Tlotleng, M., Meacock, C. et al. (2013) Deposition mechanism and microstructure of laser-assisted cold-sprayed (LACS) Al-12 wt.\% Si coatings: Effect of laser power. JOM, 65, 776-783.

12. Riveiro, A., Lusquinos, F., Comesana, R. et al. (2007) Supersonic laser spray of aluminum alloy on a ceramic substrate. Appl. Surface Sci., 254, 926-929.

13. Yuan, L.J., Luo, F., Yao, J.H. (2013) Deposition behavior at different substrate temperatures by using supersonic laser deposition. J. Iron and Steel Res. Int., 20, 87-93.

14. Tlotleng, M., Akinlabi, E., Shukla, M. et al. (2015) Microstructural and mechanical evaluation of laser-assisted cold sprayed bioceramic coatings: Potential use for biomedical applications. $J$. Thermal Spray Techn., 24, 423-435.

15. Luo, F., Cockburn, A., Cai, D.B. et al. (2015) Simulation analysis of Stellite 6 particle impact on steel substrate in supersonic laser deposition process. Ibid., 24, 378-393.

16. Yang, L.J., Li, B., Yao, J.H. et al. (2015) Effects of diamond size on the deposition characteristics and tribological behavior of diamond/Ni60 composite coating prepared by supersonic laser deposition. Diamond and Related Materials (in press). 